\title{
Insulin resistance, obesity and hypovitaminosis $D$ adversely affect pregnancy utcome: Can Supplemental vitamin D cut this risk?
}

\author{
Original \\ Article \\ Ehab Barakat ${ }^{1}$, Yehia Edris ${ }^{1}$, Adel Al-Kholy ${ }^{2}$ \\ ${ }^{1}$ Departments of Obstetrics and Gynecology, ${ }^{2}$ Medical Biochemistry, Faculty of Medicine, \\ Benha University, Benha, Egypt
}

\begin{abstract}
Aim of work: To estimate serum 25-hydroxy vitamin D (25OH-VD) and insulin, and fasting blood glucose (FBG) early in pregnancy, determine the frequency and severity of insulin resistance (IR), gestational diabetes mellitus (GDM) and pre-eclampsia (PE) during pregnancy and the impact of VD supplemental therapy (VD-ST) on these effects.

Patients and Methods: 494 pregnant women fulfilling the inclusion criteria were randomly divided into two equal groups: Study group received VD-ST as a daily oral dose of $1000 \mathrm{IU}$ soft gels with meal since 6th week till delivery, while control group did not receive VD-ST. All women gave blood samples for colorimetric estimation of FBG and ELISA estimation of serum insulin and 25-OH-VD levels. Evaluated parameters included body mass index (BMI), VD sufficiency status and homeostasis model assessment IR (HOMA-IR) score. Oral glucose tolerance test for diagnosis of GDM was performed at the 20th, 28th and 36th week GA and blood pressure was measured regularly at follow-up visits for diagnosis of PE.

Results: At time of enrolment, 405 women (81.9\%) were overweight-obese, 86 women (17.4\%) had IR and only 63 women (12.8\%) had sufficient serum 25OH-VD level. At 3rd trimester, 68 women (13.8\%) developed GDM, 71 women (14.4\%) developed PE and 23 women (4.7\%) developed both with significantly lower incidence in women who received VD-ST. Frequency of GDM and PE showed positive significant correlation with BMI and HOMA-IR score, while showed negative significant correlation with serum $25 \mathrm{OH}-\mathrm{VD}$. ROC curve analysis defined low $25 \mathrm{OH}-\mathrm{VD}$ level and high HOMA-IR score as significant sensitive predictors for development of both GDM and PE ; while receiving VD-ST was the significant specific predictor for possibility of amelioration of such event. Kaplan-Meier regression curve defined a cumulative hazard for developing both GDM and PE of $<20 \%$ with and $60 \%$ without VD-ST.

Conclusion: VD deficiency-insufficiency is a problem that requires national evaluation for predisposition and progress. The triad of maternal hypovitaminosis D, obesity and IR is associated with development of GDM and/or PE. The proposed VD supplementation regimen effectively reduced the frequencies of pregnancy-associated or induced complications; so it is effective to break that triad.
\end{abstract}

Key Words: Gestational diabetes mellitus, hypovitaminosis D, insulin resistance, pre-eclampsia, pregnancy, vitamin D supplemental therapy.

Received: 23 May 2018, Accepted: 27 June 2018

Corresponding Author: Ehab Barakat, Department of Obstetrics and Gynecology, Faculty of Medicine, Benha University, Banha, Egypt, Tel.: 01114346252,E-mail: drehabmarzouk@yahoo.com

ISSN: 2090-7265, August 2018, Vol.8, No.3

\section{INTRODUCTION}

Obesity is a worldwide epidemic with annually increasing number of overweight and obese people up to overtaking the number of malnourished ${ }^{[1]}$. Dietary energy density was directly associated with higher weight change and risk of excess adiposity ${ }^{[2]}$. Visceral adipose tissue contributed beyond overall adiposity, particularly in women (3) and is associated with multiple comorbidities $^{[4]}$.

Vitamin D (VD) is an important secosteroid hormone which after intestinal absorption was carried in blood stream bound to a binding protein to the liver to undergo hydroxylation to 25-OH VD (calcidiol) ${ }^{[5]}$. Then, calcidiol undergoes another hydroxylation in the kidneys to 1 , 25-dihydroxy-VD (calcitriol); the biologically active form of $\mathrm{VD}^{[6]}$. Biologically active VD acts through VD receptors that are widely distributed in skeletal and many nonskeletal tissues ${ }^{[7]}$ to produce a number of biological effects and therefore, contributes to human health improvement ${ }^{[8]}$.

Insulin resistance (IR) is typically defined as decreased responsiveness to insulin metabolic actions ${ }^{[9]}$, as insulin-mediated glucose disposal in skeletal muscle and adipose tissue ${ }^{[10]}$, through lower expression of glucose transporters in liver and skeletal muscle ${ }^{[11]}$ and/or disturbance in coupling of stimulation of insulin receptor by insulin ${ }^{[12]}$ ending at compensatory increase in insulin concentration despite of increased blood glucose levels ${ }^{[11]}$. IR plays a major role in pathogenesis of type 2 diabetes 
mellitus ${ }^{[13]}$, hypertension, coronary artery disease, and is tightly associated with development of obesity and its related problems ${ }^{[14]}$.

Obesity, VD deficiency and IR alone or together may play key role in the development of multiple gynecological/ obstetric diseases ${ }^{[15]}$ where the maternal-fetal unit is under the influence of vitamin $\mathrm{D}$, so breakdown in its homeostasis may underlie infertility, pre-eclampsia (PE), and gestational diabetes mellitus (GDM ${ }^{[16]}$. Women who developed insufficient $\beta$-cell compensation for pregnancyinduced IR were mostly liable to develop GDM with its related maternal and fetal complications ${ }^{[17]}$. Also, obesity and or gestational weight gain is associated with fetal large for gestational age $(\mathrm{GA})^{[18]}$, increased body fat mass especially abdominal fat ${ }^{[19]}$ and is associated with neonatal cardiometabolic makers independent of neonatal adiposity ${ }^{[20]}$.

\section{Hypothesis}

The current study hypothesized the presence of interplay between maternal obesity, IR and hypovitaminosis $\mathrm{D}$ (Hypo-VD) early in pregnancy for development of pregnancy-induced GDM and/or PE and early correction of Hypo-VD could break this vicious circle if present.

\section{Objectives}

Estimation of serum $25 \mathrm{OH}-\mathrm{VD}$ and insulin, and fasting blood glucose (FBG) early after assurance of diagnosis of pregnancy, to determine the frequency of IR, GDM and PE throughout pregnancy and the role of VD supplemental therapy (VD-ST) for lessening or ameliorating these effects.

\section{Design}

Prospective observational comparative clinical trial

\section{Setting}

Benha University Hospitals

\section{PATIENTS AND METHODS}

The study protocol was approved by the Local Ethical Committee and only women signed written fully informed consent were included in the study. All pregnant women who attended the Antenatal Outpatient Clinics for assurance of being pregnant were eligible for evaluation for study inclusion. After assuring pregnancy diagnosis, all women were asked to attend the clinic fasting on the next morning for clinical evaluation and giving fasting blood sample for laboratory investigations. Women with current DM, history of previous GDM or obesityinducing endocrinopathy, evident manifestations of hypoparathyroidism, thyrotocixosis, renal or hepatic diseases, essential hypertension and women lost during the course of pregnancy were excluded from the study.

Enrolled women were randomly allocated, using sealed envelops containing cards labeled by group title, prepared by a blinded assistant and chosen by pregnant woman herself, into two groups:

- Control group included pregnant women who will complete their pregnancy without VD-ST.

- Study group included pregnant women assigned to receive VD-ST since 6th week GA till delivery according to Grant $e t a l .^{[21]}$ in a daily oral dose of $1000 \mathrm{IU}$ soft gels to be taken with meal (Sunvite High Potency Vitamin D3 1000 IU; Puritan's Pride, Inc., Oakdale, NY, USA).

\section{Laboratory investigation}

A) Colorimetric estimation of FBG by glucose oxidase method ${ }^{[22]}$ at time of enrollment and during each follow-up visit.

B) ELISA estimation of serum 25OH-VD levels (Cayman Chemical, Ann Arbor, MI, USA) ${ }^{[23]}$ and insulin (Mercodia ELISA; ALPCO Diagnostics, Uppsala, Sweden $)^{[24]}$.

\section{Clinical Evaluation}

1. Body mass index (BMI) was calculated using the formula: weight $(\mathrm{kg}) /$ height $\left(\mathrm{m}^{2}\right)$ and patients were classified according to BMI using the World Health Organization ranges as underweight: $\mathrm{BMI}<18.5$ $\mathrm{kg} / \mathrm{m}^{2}$, normal weight: $\mathrm{BMI}=18.5-24.9 \mathrm{~kg} / \mathrm{m}^{2}$, overweight: $\mathrm{BMI}=25-29.9 \mathrm{~kg} / \mathrm{m}^{2}$ and obese: $\mathrm{BMI}=\geq 30 \mathrm{~kg} / \mathrm{m}^{2[25]}$. Obesity grades were defined after the WHO expert consultation $^{[26]}$ as BMI $<24.9$ as average, $25-<30 \mathrm{~kg} / \mathrm{m}^{2}$ as overweight, $\mathrm{BMI} \geq 30-<35 \mathrm{~kg} / \mathrm{m}^{2}$ as obese and $\mathrm{BMI} \geq 35$ $\mathrm{kg} / \mathrm{m}^{2}$ as morbid obese

2. Vitamin D sufficiency status was defined according to $25 \mathrm{OH}-\mathrm{VD}$ concentration as follows: $\geq 75 \mathrm{nmol} / \mathrm{L}$ sufficient level, $50-75 \mathrm{nmol} / \mathrm{L}$ insufficient level and $<50 \mathrm{nmol} / \mathrm{L}$ deficient level. Vitamin D deficiency was categorized as mild, moderate and severe if $25 \mathrm{OH}-\mathrm{VD}$ concentration was $25-50 \mathrm{nmol} / \mathrm{L}, 12.5-25 \mathrm{nmol} / \mathrm{L}$ and $<12.5 \mathrm{nmol} / \mathrm{L}$, respectively ${ }^{[27]}$.

3. Insulin resistance (IR) was measured by homeostasis model assessment IR (HOMA-IR) score. The HOMA-IR score was calculated as fasting serum insulin $(\mu \mathrm{U} / \mathrm{ml}) \mathrm{x}$ [FBG $(\mathrm{mg} / \mathrm{ml}) / 18]) / 22.5^{[28]}$ was considered abnormal at HOMA-IR score of $\geq 2^{[29]}$.

4. Gestational diabetes mellitus (GDM): All enrolled women were asked to fast and attend the antenatal care unit at the 20th, 28th and 36th week GA and underwent the $75 \mathrm{~g}$ Oral Glucose Tolerance Test for diagnosis of GDM according to the criteria of the International Association of Diabetes and Pregnancy Groups ${ }^{[30]}$ for abnormal OGTT, as follows: FBG $\geq 92 \mathrm{mg} / \mathrm{dl}, 1-\mathrm{h} \mathrm{BG} \geq 180 \mathrm{mg} / \mathrm{dl}$ and 2-h BG $\geq 153 \mathrm{mg} / \mathrm{dl}$.

5. Blood pressure (BP) was measured and recorded regularly at follow-up visits for diagnosis of PE which was defined as the development of hypertension after 
the 12th week GA in women who were normotensive at time of 1 st antenatal visit with systolic BP (SBP) $\geq 140$ $\mathrm{mmHg}$ and/or diastolic BP (DBP) $\geq 90 \mathrm{mmHg}$ on at least two occasions, 4 hours apart, and proteinuria (one dipstick measurement $\geq 2+$ on a voided random urine sample) $)^{[31,32]}$.

\section{Study outcomes}

1. Frequency of development of GDM and PE throughout pregnancy.

2. Relation between preliminary data and frequency of GDM and PE.

3. Effect of VD-ST on frequency and severity of GDM and PE.

\section{Statistical analysis}

Obtained data were presented as mean $\pm \mathrm{SD}$, ranges, numbers and ratios. Results were analyzed using paired t-test. Possible correlations were evaluated using Spearman's correlation coefficient and these correlations were verified as sensitivity and specificity for prediction of

Table 1: Baseline data of women enrolled in both groups development of GDM and PE using the receiver operating characteristic (ROC) curve analysis judged by the area under the curve (AUC). Statistical analysis was conducted using the SPSS (Version 15, 2006) for Windows statistical package. $P$ value $<0.05$ was considered statistically significant.

\section{RESULTS}

Throughout the study duration, 526 women were eligible for evaluation; 32 were excluded for not fulfilling the study inclusion criteria and 494 women were enrolled and randomly divided into two equal groups. At time of enrolment, calculated BMI defined 97 obese (19.6\%), 308 overweight $(62.3 \%)$ and 89 average $(18.1 \%)$ women. Estimation of FBG and serum insulin and calculation of HOMA-IR score defined 86 women $(17.4 \%)$ had IR. Estimation of serum VD defined 63 women $(12.8 \%)$ had sufficient, 142 women (28.7\%) had insufficient and 289 women $(58.5 \%)$ had deficient VD levels. There was nonsignificant $(p>0.05)$ difference between both groups as regards enrolment data as shown in table 1.

\begin{tabular}{|c|c|c|c|c|c|}
\hline Data & & & Control $(\mathrm{n}=247)$ & Study $(\mathrm{n}=247$ & $P$ value \\
\hline Age (years) & & & $33.9 \pm 2.4$ & $33.6 \pm 2.1$ & 0.978 \\
\hline \multirow[t]{4}{*}{ BMI data } & \multicolumn{2}{|c|}{ Weight (kg) } & $80 \pm 9.2$ & $81.1 \pm 6.4$ & 0.098 \\
\hline & \multicolumn{2}{|c|}{ Height (cm) } & $169.6 \pm 3.7$ & $169.4 \pm 3.7$ & 0.338 \\
\hline & & Average & $62(25.1 \%)$ & $47(19 \%)$ & 0.229 \\
\hline & BMI & Overweight & $144(58.3 \%)$ & $151(61.1 \%)$ & \\
\hline \multirow[t]{5}{*}{$\begin{array}{l}\text { Blood pressure } \\
\text { measures }(\mathrm{mmHg})\end{array}$} & & Obese & $41(16.6 \%)$ & $49(19.9 \%)$ & 0.059 \\
\hline & \multicolumn{2}{|c|}{ BMI (kg/m2) } & $27.8 \pm 3.1$ & $28.3 \pm 2.5$ & \\
\hline & \multicolumn{2}{|c|}{ Systolic } & $118.3 \pm 3.8$ & $118 \pm 4.6$ & 0.863 \\
\hline & \multicolumn{2}{|c|}{ Diastolic } & $82.5 \pm 4.3$ & $83.6 \pm 3.1$ & 0.627 \\
\hline & \multicolumn{2}{|c|}{ FBG (mg/dl) } & $118.9 \pm 11$ & $120.9 \pm 12.2$ & 0.068 \\
\hline \multirow{6}{*}{$\begin{array}{ll}\text { Insulin } & \text { resistance } \\
\text { (IR) data } & \end{array}$} & \multicolumn{2}{|c|}{ Serum insulin } & $4.85 \pm 1.3$ & $4.7 \pm 1.2$ & 0.597 \\
\hline & \multirow{2}{*}{ IR } & Yes & $45(18.2 \%)$ & $41(16.6 \%)$ & \multirow{2}{*}{0.635} \\
\hline & & No & $202(81.8 \%)$ & $206(83.4 \%)$ & \\
\hline & \multicolumn{2}{|c|}{ HOMA-IR score } & $1.44 \pm 0.41$ & $1.45 \pm 0.46$ & 0.843 \\
\hline & \multicolumn{2}{|c|}{ Sufficient } & $31(12.7 \%)$ & $32(13 \%)$ & \\
\hline & \multicolumn{2}{|c|}{ Insufficient } & $68(27.5 \%)$ & $74(30 \%)$ & \\
\hline \multirow{4}{*}{ VD sufficiency data } & \multirow{4}{*}{ Deficient } & Mild & $92(37.2 \%)$ & $85(34.4 \%)$ & 0.712 \\
\hline & & Moderate & $49(19.8 \%)$ & $44(17.8 \%)$ & \\
\hline & & Severe & $7(2.8 \%)$ & $12(4.8 \%)$ & \\
\hline & & & $45.8 \pm 20.3$ & $46.3 \pm 20.8$ & 0.798 \\
\hline
\end{tabular}

Data are presented as mean $\pm \mathrm{SD}$ and numbers; percentages are in parenthesis; $\mathrm{BMI}$ : Body mass 
At the start of 3rd trimester, all women had higher FBG and serum insulin levels with higher HOMA-IR score compared to their respective baseline measures. The 3 rd trimester measures were significantly $(p<0.05)$ higher in control women compared to their baseline measures and to 3rd trimester measures of study women. On the other hand, FBG and calculated HOMA-IR score were significantly higher, while serum insulin levels were nonsignificantly higher in study women compared to their baseline measures. As regards IR, at the 3rd trimester, 166 women (33.6\%) were IR; 113 in control and 53 women in study group with significantly $(p<0.05)$ higher difference in frequency of IR between both groups and in control group versus its 12 th wk frequency. Moreover, at the start of 3rd trimester, 68 women (13.8\%) developed GDM; 45 $(9.3 \%)$ and $23(9.3 \%)$ women in control and study groups, respectively with significantly $(p=0.004)$ lower difference in favor of study group (Table 2).

Table 2: Glucogenic data detected at 3rd trimester compared to at the 12th wk GA in both groups

\begin{tabular}{|c|c|c|c|c|c|c|c|}
\hline \multicolumn{2}{|c|}{ Group } & \multicolumn{3}{|c|}{ Control } & \multicolumn{3}{|c|}{ Study } \\
\hline Parameter & Time & 12th wk GA & 3rd trimester & $P$ value & 12th wk GA & 3rd trimester & $P=$ \\
\hline FBG & & $118.9 \pm 11$ & $136.7 \pm 9.8$ & 0.0001 & $120.9 \pm 12.2$ & $124.3 \pm 23^{*}$ & 0.001 \\
\hline Insulin & & $4.85 \pm 1.3$ & $5.85 \pm 1.32$ & $<0.001$ & $4.7 \pm 1.2$ & $4.98 \pm 1.19^{*}$ & 0.248 \\
\hline \multirow[t]{2}{*}{ HOMA-IR score } & Frequency & $\begin{array}{c}45(18.2 \%) \\
202(81.8 \%)\end{array}$ & $\begin{array}{l}113(45.7 \%) \\
134(54.3 \%)\end{array}$ & $<0.001$ & $\begin{array}{l}41(16.6 \%) \\
206(83.4 \%)\end{array}$ & $\begin{array}{l}53 *(23.9 \%) \\
194(76.1 \%)\end{array}$ & 0.169 \\
\hline & Score & $1.44 \pm 0.41$ & $1.98 \pm 0.5$ & $<0.001$ & $1.47 \pm 0.5$ & $1.55 \pm 0.6^{*}$ & 0.019 \\
\hline \multirow{2}{*}{ GDM } & $\mathrm{Y}$ & 0 & 45 (18.2\%) & & 0 & $23(9.3 \%)$ & \\
\hline & $\mathrm{N}$ & 0 & $202(81.8 \%)$ & & 0 & $224(90.7 \%)$ & \\
\hline
\end{tabular}

Data are presented as mean \pm SD and numbers; percentages are in parenthesis; FBG: Fasting blood glucose; HOMA-IR: Homeostasis model assessment of insulin resistance; IR; GDM: Gestational diabetes mellitus

Furthermore, at the start of 3rd trimester, all women got increased blood pressure measures. SBP measures were significantly higher, while DBP measures were non-significantly higher in both groups compared to their baseline measures with significantly higher measures in control versus study women. Seventy-one women developed high blood pressure measures up to levels diagnosed as PE; 46 in control and 25 in study group with significantly higher frequency among women of study women compared to their baseline measures (Table 3). Twenty-three women (4.7\%) developed both GDM and PE; 16 in control and 7 in study groups with significantly lower incidence of both GDM and PE among study versus control women.

Table 3: Blood pressure data estimated at 3rd trimester compared to at the 12th wk GA in both groups

\begin{tabular}{|c|c|c|c|c|c|c|c|}
\hline \multicolumn{2}{|l|}{ Group } & \multicolumn{3}{|c|}{ Control } & \multicolumn{3}{|c|}{ Study } \\
\hline Parameter & Time & 12th wk GA & 3rd trimester & $\mathrm{P}$ value & 12th wk GA & 3rd trimester & $P$ value \\
\hline SBP (mmHg) & & $118.3 \pm 3.8$ & $123.2 \pm 12.5^{*}$ & 0.009 & $118 \pm 4.6$ & $121.6 \pm 13.9$ & 0.039 \\
\hline DBP (mmHg) & & $82.5 \pm 4.3$ & $85.6 \pm 6.9$ & 0.078 & $83.6 \pm 3.1$ & $84.1 \pm 8.5$ & 0.078 \\
\hline
\end{tabular}

Data are presented as mean \pm SD and numbers; percentages are in parenthesis; SBP: Systolic blood pressure; DBP: Diastolic blood pressure

Development of GDM showed positive significant correlation with BMI and HOMA-IR score, while showed negative significant correlation with serum 25OHD. Similarly, development of PE were positively correlated with BMI and FBG at 3rd trimester and negatively correlated with serum 25-OHD. Moreover, development of both GDM and PE positively correlated with BMI, FBG at 3rd trimester, HOMA-IR score and negatively correlated with serum 25OHD (Table 4). 
Table 4: Correlation of the development of GDM or PE in the 3rd trimester with BMI, HOMA-IR score and serum 25-OHD

\begin{tabular}{lcccccccc}
\hline & \multicolumn{2}{c}{ BMI } & & \multicolumn{2}{c}{ FBG at 3rd trimester } & \multicolumn{2}{c}{ HOMA-IR score } & \multicolumn{2}{c}{ Serum 25-OHD } \\
\hline \multirow{2}{*}{ GDM } & Rho & $P$ & Rho & $P$ & Rho & $P$ & Rho & $P$ \\
PE & 0.171 & $<0.001$ & 0.533 & $<0.004$ & 0.149 & $<0.001$ & -0.211 & $<0.001$ \\
Both & 0.190 & $<0.001$ & 0.107 & $=0.017$ & 0.061 & 0.177 & -0.219 & $<0.001$ \\
& 0.219 & $<0.001$ & 0.388 & $<0.001$ & 0.131 & 0.004 & -0.248 & $<0.001$ \\
\hline
\end{tabular}

Rho: Spearman's correlation coefficient; BMI: Body mass index; FBG: Fasting blood glucose; HOMA-IR: Homeostasis model assessment of insulin resistance; IR; 25-OHD: 25-hydroxy vitamin D; GDM: Gestational diabetes mellitus

ROC curve analysis of clinical and laboratory data of enrolled women as predictors for development of both GDM and PE defined Hypo-VD as the significant sensitive predictor for such event followed by high HOMA-IR score and FBG at the 3rd trimester, while receiving VD-
ST was the significant specific predictor for possibility of amelioration or prevention of such event (Fig. 1). KaplanMeier Regression curve defined a cumulative hazard for developing both GDM and PE of $<20 \%$ with and $60 \%$ without VD-ST (Fig. 2).

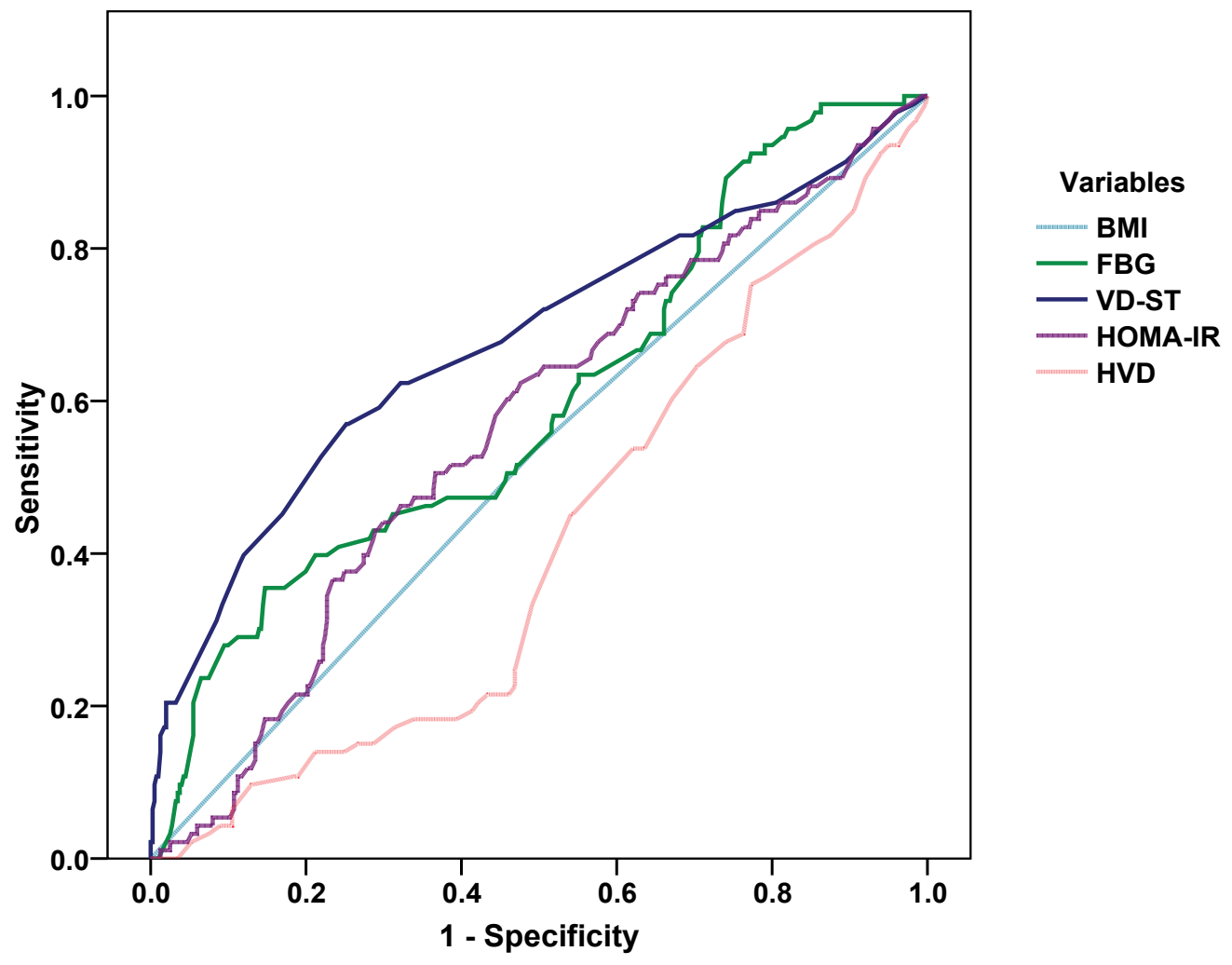

Fig. 1: ROC curve analysis for variable as predictors for development of both GDM and PE 


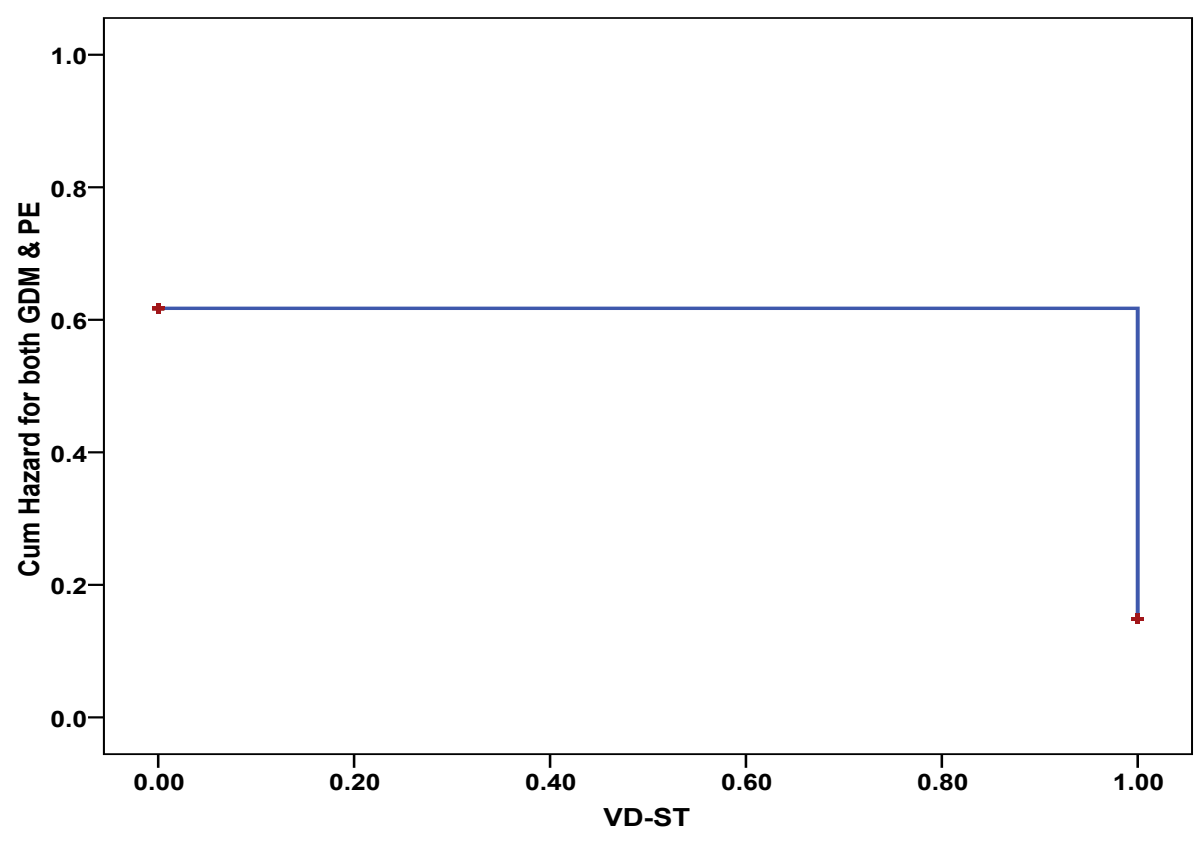

Fig. 2: Kaplan-Meier Regression curve for cumulative hazard for developing both GDM and PE with and without VD-ST

\section{DISCUSSION}

The current observational study provided multiple interesting findings; firstly out of the studied women, when getting pregnant, only $19.6 \%$ have average weight, $17.4 \%$ were insulin resistant and $58.5 \%$ had hypovitaminosis D (Hypo-VD). These figures point to the fact that high percentage of studied population had pre-pregnancy obesity and Hypo-VD and may be insulin resistant, so they could be mostly considered as having pre-pregnancy risk for committing pregnancy-induced or -associated morbidities that may affect pregnancy outcome.

The reported figure of Hypo-VD goes in hand with Loy et al. ${ }^{[33]}$ who reported VD inadequacy rate of $41.3 \%$, and Al-Shaikh et al.$^{[34]}$ who reported VD deficiency in $86.4 \%$ of pregnant females in Riyadh and GDM was the commonest complication detected in $11.1 \%$. Moreover, Karras et al. ${ }^{[35]}$ out of literature systemic review found the prevalence of VD insufficiency among pregnant Greece women ranged from 9.3 to $41.4 \%$, whereas that of VD deficiency from 22.7 to $90.3 \%$.

Furthermore, Naseh et al. ${ }^{[36]}$ and Kilicaslan et al. ${ }^{[37]}$ detected VD deficiency rates of $27 \%$ and $53 \%$ and insufficiency rates of $73 \%$ and $47 \%$, respectively, in their series of pregnant women. Moreover, Wierzejska et al. ${ }^{[38]}$ detected higher VD levels in maternal and umbilical cord blood in summer than in winter, but only $16 \%$ of studied pregnant women had the optimal VD level during summer. Recently, in 2018; Gustafsson et al. ${ }^{[39]}$ reported decreased $1,25(\mathrm{OH}) 2 \mathrm{D}$ in $45 \%$ of pregnant women with significant difference than women with adequate vitamin $\mathrm{D}$ status and found no association of VD indices and parathyroid hormone changes during pregnancy.
Secondly, pregnancy itself is a diabetogenic process as evidenced by the increased levels of FBG in all patients, increased frequency of IR women and higher HOMAIR score at the 3rd trimester compared to the 12th wk GA. Moreover, 68 women developed GDM throughout pregnancy course for a frequency of $13.8 \%$. Moreover, throughout the duration of pregnancy, 71 women developed PE despite being normotensive at the 12th wk GA for a frequency of $14.4 \%$ and at the start of 3rd trimester, all patients showed increased blood pressure measures, irrespective of progressing to PE or not. Twenty-three women developed both GDM and PE with significantly lower incidence in women received VD-ST.

Thirdly, low 25OH-VD level showed positive significant correlation, while VD-ST showed negative significant correlation with a development of PE and GDM and with IR. These findings are in accordance with Lacroix et al. ${ }^{[40]}$ who detected higher risk of GDM in women had lower 1 st trimester 25OHD levels and Lu et al. ${ }^{[41]}$ who documented that maternal vitamin $\mathrm{D}$ insufficiency is associated with increased risk of GDM. Moreover, AlShaikh et al. ${ }^{[34]}$ documented that GDM was the commonest complication encountered among pregnant women who had vitamin D deficiency for a frequency of $11.1 \%$ and DeRegil et al. ${ }^{[42]}$ found $7.5 \%$ and $6.5 \%$ of pregnant women developed PE and GDM, respectively. In line with detected correlations, Jafarzadeh et al..$^{[43]}$, Dodds et $a l^{[44]}$ and Triunfo et al. ${ }^{[45]}$ reported that in pregnant women, lower 25OHD concentrations showed an inverse association with maternal glycaemia and increased risk of GDM. Also, Naseh et al. ${ }^{[46]}$ found that maternal weight before delivery negatively correlated with maternal serum VD level.

The obtained data concerning VD-ST point to the role played by its deficiency or insufficiency in the 
development of maternal pregnancy-induced or-associated complications and provide a guideline for its use as a prepregnancy prophylactic therapy. In line with the reported beneficial effect of VD-ST, in 2018; Rostami et al. ${ }^{[47]}$ found VD-ST improved serum 25(OH)D levels and decreased adverse pregnancy outcomes including PE, GDM and preterm delivery by 60,50 and $40 \%$ than in women maintained without of VD-ST. Also, Shu and Huang ${ }^{[48]}$ detected a significant decrease in peripheral systolic and diastolic blood pressure in hypertensive patients after 8 weeks of VD supplementation. Moreover, Wilson et al. (2018) found that the risk of GDM was reduced by $53 \%$ with high $(>81 \mathrm{nmol} / \mathrm{L})$ VD status when compared to moderate-high and Zhang et al. ${ }^{[50]}$ reported that that low blood VD level could increase the risk of GDM, and VD supplementation during pregnancy could ameliorate the condition of GDM.

VD-ST was provided as single daily dose of $1000 \mathrm{IU}$, this supplementation regimen is in accordance with Cooper et al. ${ }^{[51]}$ who found VD supplementation with $1000 \mathrm{IU} /$ day during pregnancy is safe and sufficient to ensure replete VD in most of the pregnant women and with Yilmaz et al. ${ }^{[52]}$ who documented that VD supplementation by $1200 \mathrm{IU} /$ day since the 12th gestational week can prevent neonatal VD deficiency.

\section{CONCLUSION}

The obtained results and review of literature allowed concluding that VD deficiency-insufficiency is a settled problem that requires national evaluation for predisposition and progress. Maternal hypovitaminosis D, obesity and insulin resistance constitute a triad that deleteriously affects pregnant women and is associated with development of GDM and/or PE. The proposed VD supplementation regimen effectively reduced the frequencies of pregnancyassociated or induced complications; so it is effective to break that triad. Wider scale studies are mandatory to evaluate the effect of pre-gestational VD-ST on oncoming pregnancy and the effect of injectable forms of VD that may be more convenient for patients.

\section{CONFLICT OF INTEREST}

There are no conflicts of interest.

\section{REFERENCES}

1. Demerath EW, Reed D, Choh AC, Soloway L, Lee M, Czerwinski SA, Chumlea WC, Siervogel RM, Towne B: Rapid postnatal weight gain and visceral adiposity in adulthood: the Fels Longitudinal Study. Obesity (Silver Spring). 2009; 17(11): 2060-6.

2. Rouhani MH, Haghighatdoost F, Surkan PJ,
Azadbakht L: Associations between dietary energy density and obesity: A systematic review and meta-analysis of observational studies. Nutrition. 2016; 32(10): 1037-47.

3. Gast KB, den Heijer M, Smit JW, Widya RL, Lamb HJ, de Roos A, Jukema JW, Rosendaal FR, de Mutsert R; NEO study group: Individual contributions of visceral fat and total body fat to subclinical atherosclerosis: The NEO study. Atherosclerosis. 2015; 241(2): 547-54.

4. Wang H, Chen YE, Eitzman DT: Imaging body fat: techniques and cardiometabolic implications. Arterioscler Thromb Vasc Biol. 2014; 34(10): 2217-23.

5. Lapillonne A: Vitamin D, deficiency during pregnancy may impair maternal and fetal outcomes. Med Hypotheses 2010, 74(1): 71-5.

6. Cho GJ, Hong SC, Oh MJ, Kim HJ: Vitamin D deficiency in gestational diabetes mellitus and the role of the placenta. Am J Obstet Gynecol. 2013; 209(6): 560.e1-8.

7. Yoon HK: Gestational Diabetes Mellitus, Fetal Growth and Vitamin D. J Bone Metab. 2017;24(3): 155-59.

8. Teegarden D, Donkin SS: Vitamin D: emerging new roles in insulin sensitivity. Nutr Res Rev. 2009; 22(1): 82-92.

9. Muniyappa R, Lee S, Chen H, Quon MJ: Current approaches for assessing insulin sensitivity and resistance in vivo: advantages, limitations, and appropriate usage. Am J Physiol Endocrinol Metab.; 294:E15-E26, 2008.

10. Gutch M, Kumar S, Razi SM, Gupta KK, Gupta A: Assessment of insulin sensitivity/resistance. Indian J Endocrinol Metab. 2015; 19(1): 160-4.

11. Chen L, Xu WM, Zhang D: Association of abdominal obesity, insulin resistance, and oxidative stress in adipose tissue in women with polycystic ovary syndrome. Fertil Steril. 2014; 102(4): 1167-1174.

12. Baillargeon JP, Iuorno MJ, Apridonidze T, Nestler JE: Uncoupling between insulin and release of a D-chiro-inositol-containing inositolphosphoglycan mediator of insulin action in obese women With polycystic ovary syndrome. Metab Syndr Relat Disord. 2010; 8(2): 127-36.

13. Macauley M, Smith FE, Thelwall PE, Hollingsworth KG, Taylor R: Diurnal variation 
in skeletal muscle and liver glycogen in humans with normal health and Type 2 diabetes. Clin Sci (Lond). 2015; 128(10): 707-13.

14. Müller MJ, Lagerpusch M, Enderle J, Schautz B, Heller M, Bosy-Westphal A: Beyond the body mass index: tracking body composition in the pathogenesis of obesity and the metabolic syndrome. Obes Rev. 2012; 13 Suppl 2: 6-13.

15. Colonese F, Laganà AS, Colonese E, Sofo V, Salmeri FM, Granese R, Triolo O: The pleiotropic effects of vitamin D in gynaecological and obstetric diseases: an overview on a hot topic. Biomed Res Int. 2015; 2015:986281.

16. Nandi A, Sinha N, Ong E, Sonmez H, Poretsky L: Is there a role for vitamin $\mathrm{D}$ in human reproduction? Horm Mol Biol Clin Investig. 2016; 25(1): 15-28.

17. Retnakaran R, Ye C, Kramer CK, Connelly PW, Hanley AJ, Sermer M, Zinman B: Evaluation of Circulating Determinants of Beta-Cell Function in Women With and Without Gestational Diabetes. J Clin Endocrinol Metab. 2016; 101(7): 2683-91.

18. Wong T, Barnes RA, Ross GP, Cheung NW, Flack JR: Are the Institute of Medicine weight gain targets applicable in women with gestational diabetes mellitus? Diabetologia. 2017; 60(3): 416423.

19. Carlsen EM, Renault KM, Nørgaard K, Nilas L, Jensen JE, Hyldstrup L, Michaelsen KF, Cortes D, Pryds O: Newborn regional body composition is influenced by maternal obesity, gestational weight gain and the birthweight standard score. Acta Paediatr. 2014; 103(9): 939-45.

20. Lemas DJ, Brinton JT, Shapiro AL, Glueck DH, Friedman JE, Dabelea D: Associations of maternal weight status prior and during pregnancy with neonatal cardiometabolic markers at birth: the Healthy Start study. Int J Obes (Lond). 2015; 39(10): 1437-42.

21. Grant CC, Stewart AW, Scragg R, Milne T, Rowden J, Ekeroma A, Wall C, Mitchell EA, Crengle S, Trenholme A, Crane J, Camargo CA $\mathrm{Jr}$ : Vitamin $\mathrm{D}$ during pregnancy and infancy and infant serum 25-hydroxyvitamin D concentration. Pediatrics. 2014; 133(1): e143-53

22. Tinder P: Determination of blood glucose. Ann. Clin. Biochem.; 6:24, 1969.

23. Andersen L, Dinesen B, Jørgensen PN, Poulsen $F$ and Røder ME: Enzyme immunoassay for intact human insulin in serum or plasma. Clinical Chemistry, 1993; 38: 578-82.
24. Gordon C, Yates AP, Davies D: Evidence for a direct action of exogenous insulin on the pancreatic islets of diabetic mice: islet response to insulin pre-incubation. Diabetologia, 1985; 28, 291-4.

25. Bray GA: Pathophysiology of obesity. Am J Clin Nutr, 1992; 55: 488S-94S.

26. WHO expert consultation. Appropriate Body-mass index for Asian populations and its implications for policy and intervention strategies. Lancet, 2004; 157-63.

27. Stroud ML, Stilgoe S, Stott VE, Alhabian O, Salman K: Vitamin D - a review. Aust Fam Physic ian. 2008; 37(12):

1002-5.

28. Matthews DR, Hosker J, Rudenski A, Naylor B, Treacher D, Turner R: Homeostasis model assessment: insulin resistance and beta-cell function from fasting plasma glucose and insulin concentrations in man. Diabetologia, 1985; 28: 412-9.

29. Ascaso JF, Romero P, Real JT, Priego A, Valdecabres C, Carmena R: Insulin resistance quantification by fasting insulin plasma values and HOMA index in a non-diabetic population. Med Clin (Barc), 2001; 117: 530-3.

30. International association of diabetes and pregnancy study groups (IADPSG) recommendations on the diagnosis and classification of hyperglycemia in pregnancy. Diabetes Care. 2010; 33: 676-82.

31. Gifford RW, August PA, Cunningham FG: Report of the national high blood pressure working group on research on hypertension in pregnancy. Am. J Obstet. Gynecol., 2000; 183: S1-22.

32. Lenfant C; National Education Program Working Group on High Blood Pressure in Pregnancy: Working group report on high blood pressure in pregnancy. J Clin Hypertens (Greenwich). 2001; 3(2): 75-88

33. Loy SL, Lek N, Yap F, Soh SE, Padmapriya N, Tan KH, Biswas A, Yeo GS, Kwek K, Gluckman PD, Godfrey KM, Saw SM, Müller-Riemenschneider F, Chong YS, Chong MF, Chan JK; Growing Up in Singapore Towards Healthy Outcomes (GUSTO) study group: Association of Maternal Vitamin D Status with Glucose Tolerance and Caesarean Section in a Multi-Ethnic Asian Cohort: The Growing Up in Singapore Towards Healthy Outcomes Study. PLoS One. 2015; 10(11): e0142239.

34. Al-Shaikh GK, Ibrahim GH, Fayed AA, Al- 
Mandeel H: Impact of vitamin D deficiency on maternal and birth outcomes in the Saudi population: a cross-sectional study. BMC Pregnancy Childbirth. 2016; 16: 119.

35. Karras S, Paschou SA, Kandaraki E, Anagnostis P, Annweiler C, Tarlatzis BC, Hollis BW, Grant WB, Goulis DG: Hypovitaminosis D in pregnancy in the Mediterranean region: a systematic review. Eur J Clin Nutr. 2016;70(9): 979-86.

36. Naseh A, Ashrafzadeh S, Rassi S: Prevalence of vitamin D deficiency in pregnant mothers in Tehran and investigating its association with serum glucose and insulin. J Matern Fetal Neonatal Med. 2017: 1-7.

37. Kılicaslan AÖ, Kutlu R, Kilinc I, Ozberk DI: The effects of vitamin $\mathrm{D}$ supplementation during pregnancy and maternal vitamin $\mathrm{D}$ levels on neonatal vitamin $\mathrm{D}$ levels and birth parameters. J Matern Fetal Neonatal Med. 2017: 1-8.

38. Wierzejska R, Jarosz M, Sawicki W, Bachanek M, Siuba-Strzelińska M: Vitamin D Concentration in Maternal and Umbilical Cord Blood by Season. Int J Environ Res Public Health. 2017; 14(10). pii: E1121.

39. Gustafsson MK, Romundstad PR, Stafne SN, Helvik AS, Stunes AK, Mørkved S, Salvesen $\mathrm{K} \AA$, Thorsby PM, Syversen U: Alterations in the vitamin $\mathrm{D}$ endocrine system during pregnancy: A longitudinal study of 855 healthy Norwegian women. PLoS One. 2018;13(4): e0195041.
40. Lacroix M, Battista MC, Doyon M, Houde G, Ménard J, Ardilouze JL, Hivert MF, Perron P: Lower vitamin D levels at first trimester are associated with higher risk of developing gestational diabetes mellitus. Acta Diabetol. 2014; 51(4): 609-16.

41. Lu M, Xu Y, Lv L, Zhang M: Association between vitamin $\mathrm{D}$ status and the risk of gestational diabetes mellitus: a meta-analysis. Arch Gynecol Obstet. 2016; 293(5): 959-66.

42. De-Regil LM, Palacios C, Lombardo LK, PeñaRosas JP: Vitamin D supplementation for women during pregnancy. Sao Paulo Med J. 2016; 134(3): 274-5.

43. Jafarzadeh L, Motamedi A, Behradmanesh M, Hashemi R: A comparison of serum levels of 25-hydroxy vitamin $\mathrm{d}$ in pregnant women at risk for gestational diabetes mellitus and women without risk factors. Mater Sociomed. 2015; 27(5): 318-22.

44. Dodds L, Woolcott CG, Weiler H, Spencer A, Forest JC, Armson BA, Giguère Y: Vitamin D Status and Gestational Diabetes: Effect of Smoking Status during Pregnancy. Paediatr Perinat Epidemiol. 2016; 30(3): 229-37

45. Triunfo S, Lanzone A, Lindqvist PG: Low maternal circulating levels of vitamin $\mathrm{D}$ as potential determinant in the development of gestational diabetes mellitus. J Endocrinol Invest. 2017. doi: 10.1007/s40618-017-0696-9. 